Supporting Information

\title{
Identification and Quantitation of Taste-Active \\ Compounds in Dried Scallops by Combined Application of the Sensomics- and a quantitative NMR Approach
}

\author{
Sebastian Dirndorfer ${ }^{\dagger}$ §, Richard Hammerl ${ }^{\dagger}$, Seiji Kitajima ${ }^{\ddagger}$, Ryo Kitada ${ }^{\ddagger}$, Oliver \\ Frank $^{\dagger}$, Andreas Dunkel ${ }^{\#}$ and Thomas Hofmann ${ }^{\dagger, ~ * ~}$ \\ tChair of Food Chemistry and Molecular and Sensory Science, Technical \\ University of Munich, Lise-Meitner-Str. 34, D-85354 Freising, Germany and
} \#Leibniz-Institute for Food Systems Biology at the Technical University of Munich, Lise-Meitner-Str. 34, D-85354 Freising, Germany

ҒInstitute of Food Sciences \& Technologies, Ajinomoto Co., Inc., 1-1 Suzuki-cho, Kawasaki-ku, Kawasaki, Kanagawa 210-8681, Japan

§current address: Leibniz-Institute for Food Systems Biology at the Technical University of Munich, Lise-Meitner-Str. 34, D-85354 Freising, Germany

Running Title: Identification and Quantitation of Taste-Active Compounds in Dried Scallops by Combined Application of the Sensomics- and a quantitative NMR Approach

\section{* To whom correspondence should be addressed}

PHONE +49-8161-712902

FAX +49-8161-712949

E-MAIL thomas.hofmann@tum.de 
Figure Captions

Figure S1. ${ }^{1} \mathrm{H}$ NMR spectrum of octopine (1) $\left(\mathrm{D}_{2} \mathrm{O}, 400 \mathrm{MHz}\right)$.

Figure S2. ${ }^{1} \mathrm{H}$ NMR spectrum of octopine- ${ }^{13} \mathrm{C}_{6}\left(1-{ }^{13} \mathrm{C}_{6}\right)\left(\mathrm{D}_{2} \mathrm{O}, 400 \mathrm{MHz}\right)$.

Figure S3. $\quad{ }^{1} \mathrm{H}$ NMR spectrum of alanopine $(2)\left(\mathrm{D}_{2} \mathrm{O}, 400 \mathrm{MHz}\right)$.

Figure S4. $\quad{ }^{1} \mathrm{H}$ NMR spectrum of alanopine- ${ }^{13} \mathrm{C}_{3}\left(2^{-13} \mathrm{C}_{3}\right)\left(\mathrm{D}_{2} \mathrm{O}, 400 \mathrm{MHz}\right)$.

Figure S5. $\quad{ }^{1} \mathrm{H}$ NMR spectrum of strombine $(3)\left(\mathrm{D}_{2} \mathrm{O}, 400 \mathrm{MHz}\right)$.

Figure S6. ${ }^{1} \mathrm{H}$ NMR spectrum of strombine- $\mathrm{d}_{2}\left(3-\mathrm{d}_{2}\right)\left(\mathrm{D}_{2} \mathrm{O}, 400 \mathrm{MHz}\right)$.

Figure S7. ${ }^{1} \mathrm{H}$ NMR spectrum of tauropine $(4)\left(\mathrm{D}_{2} \mathrm{O}, 400 \mathrm{MHz}\right)$.

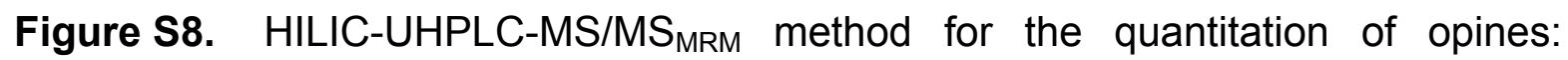
development of chromatographic separation 
Figure S1 (Dirndorfer et al.)

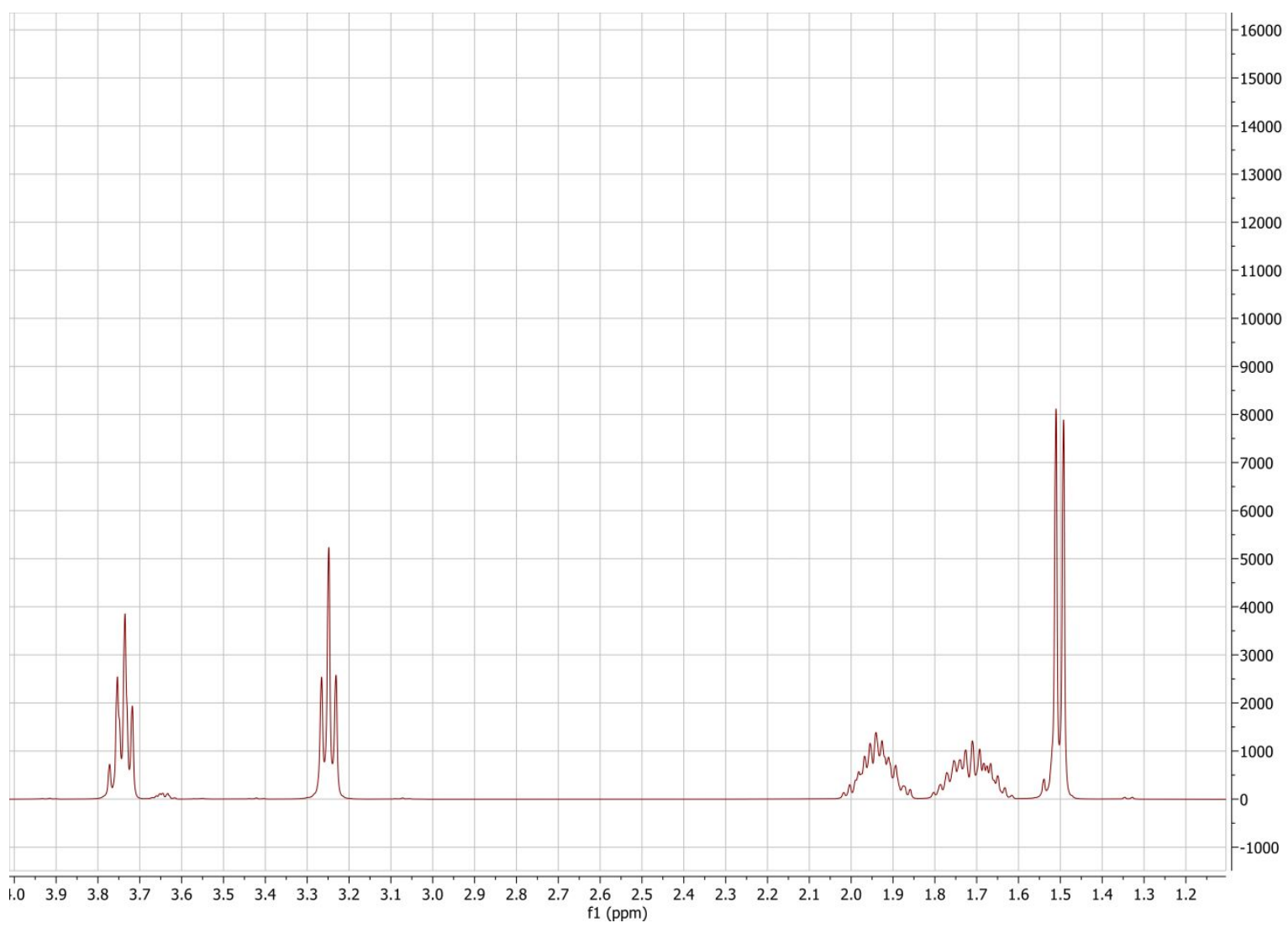

46 
Figure S2 (Dirndorfer et al.)

48

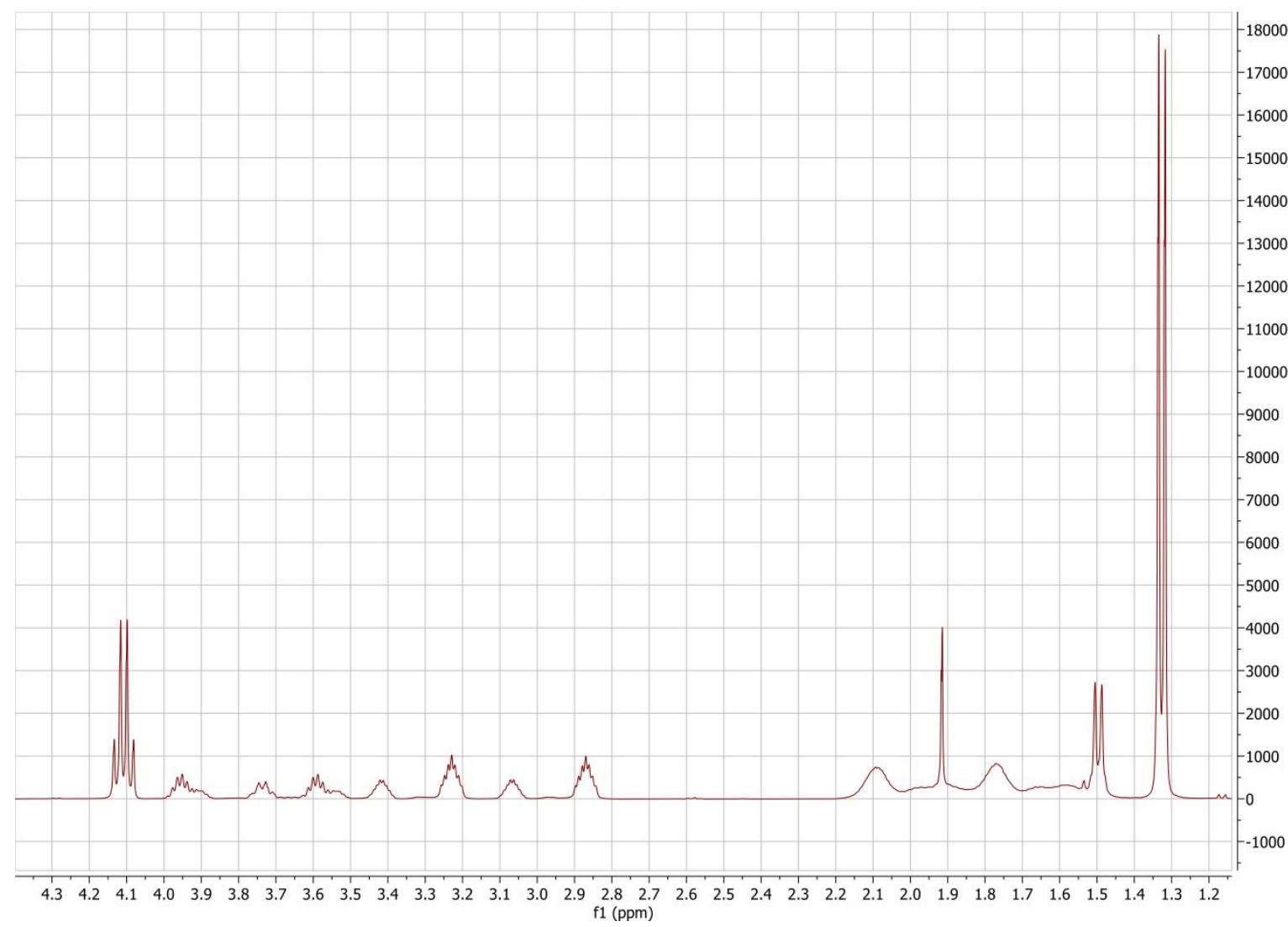

49

50 
Figure S3 (Dirndorfer et al.)

52

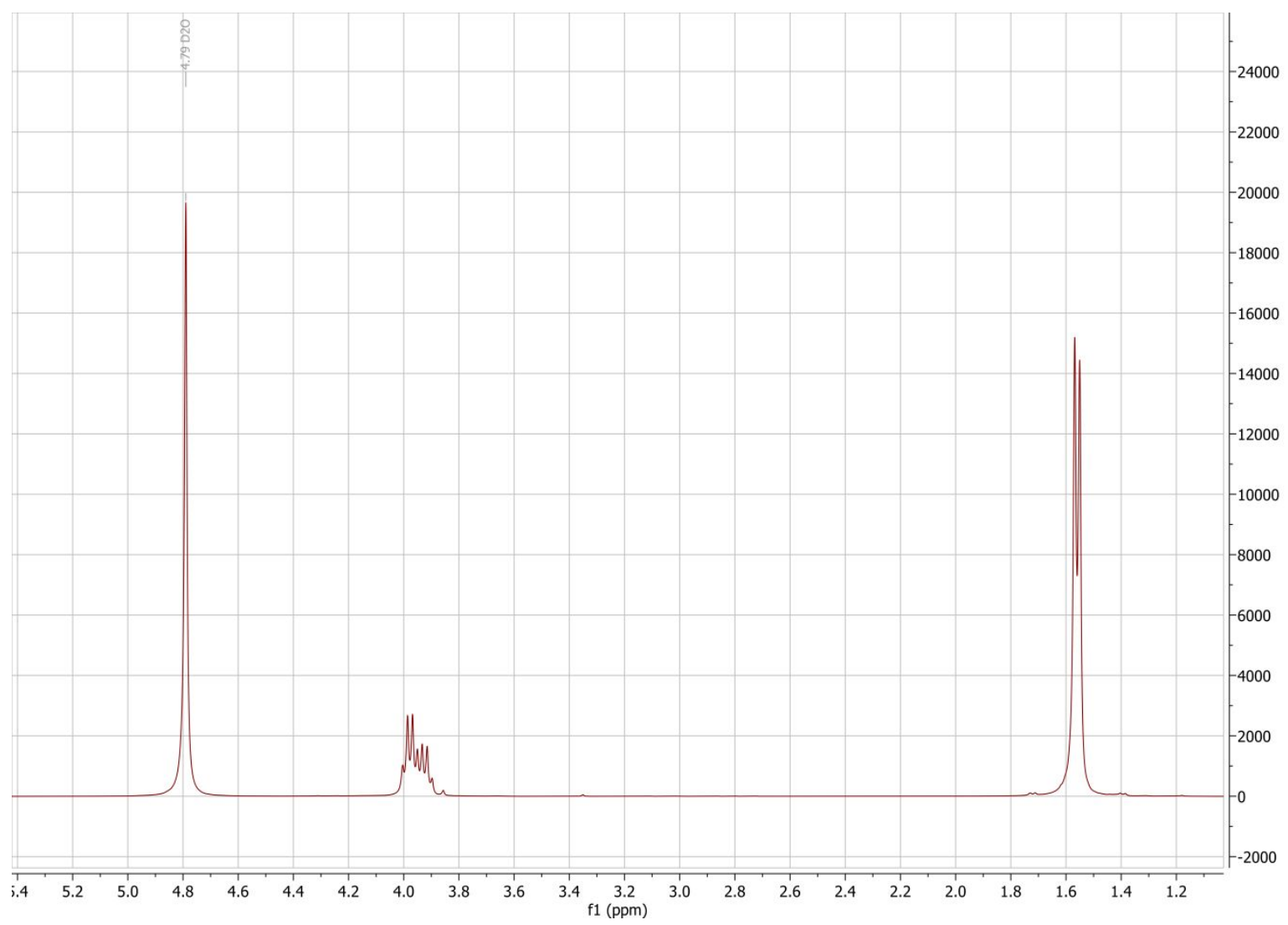

53 
Figure S4 (Dirndorfer et al.)

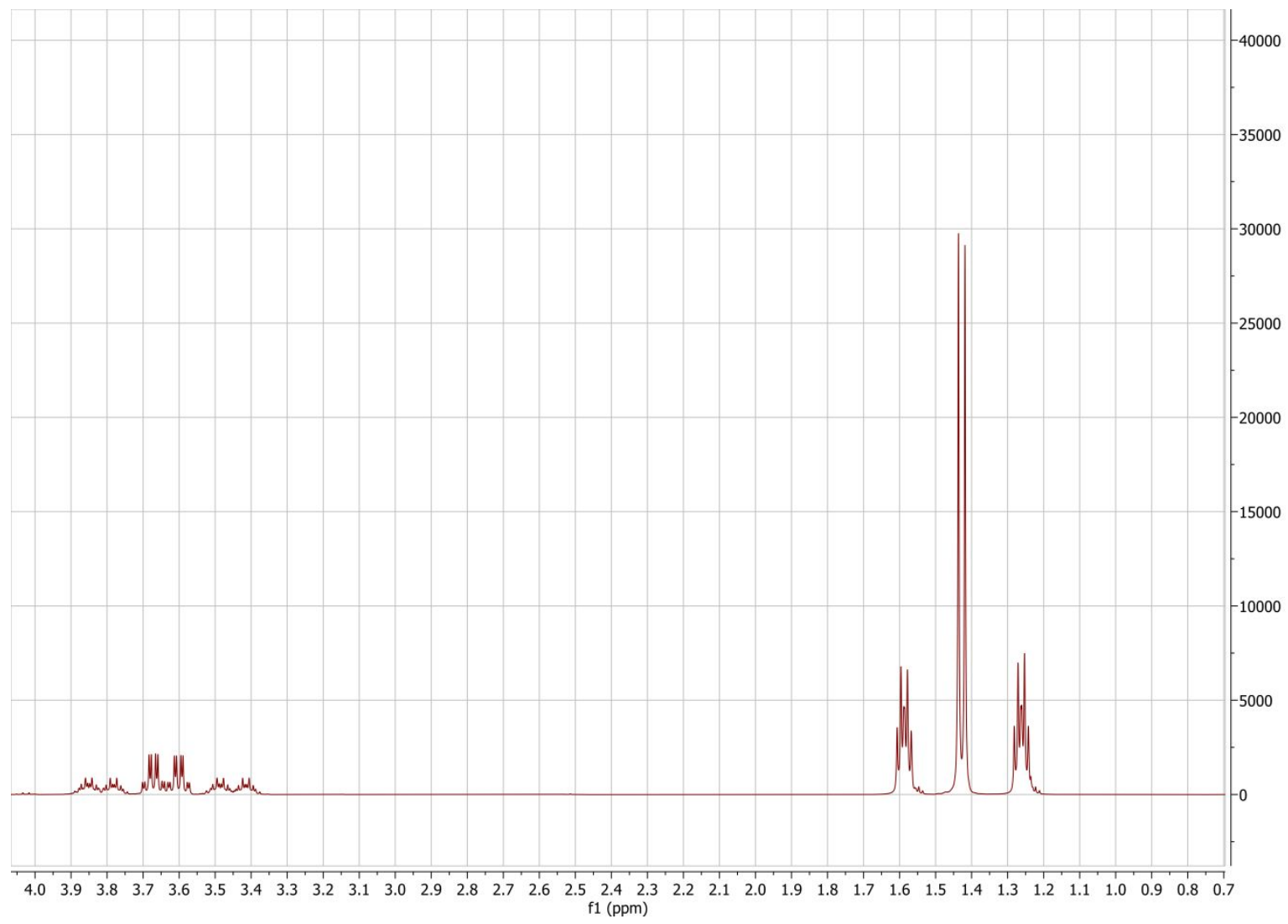

56 
Figure S5 (Dirndorfer et al.)

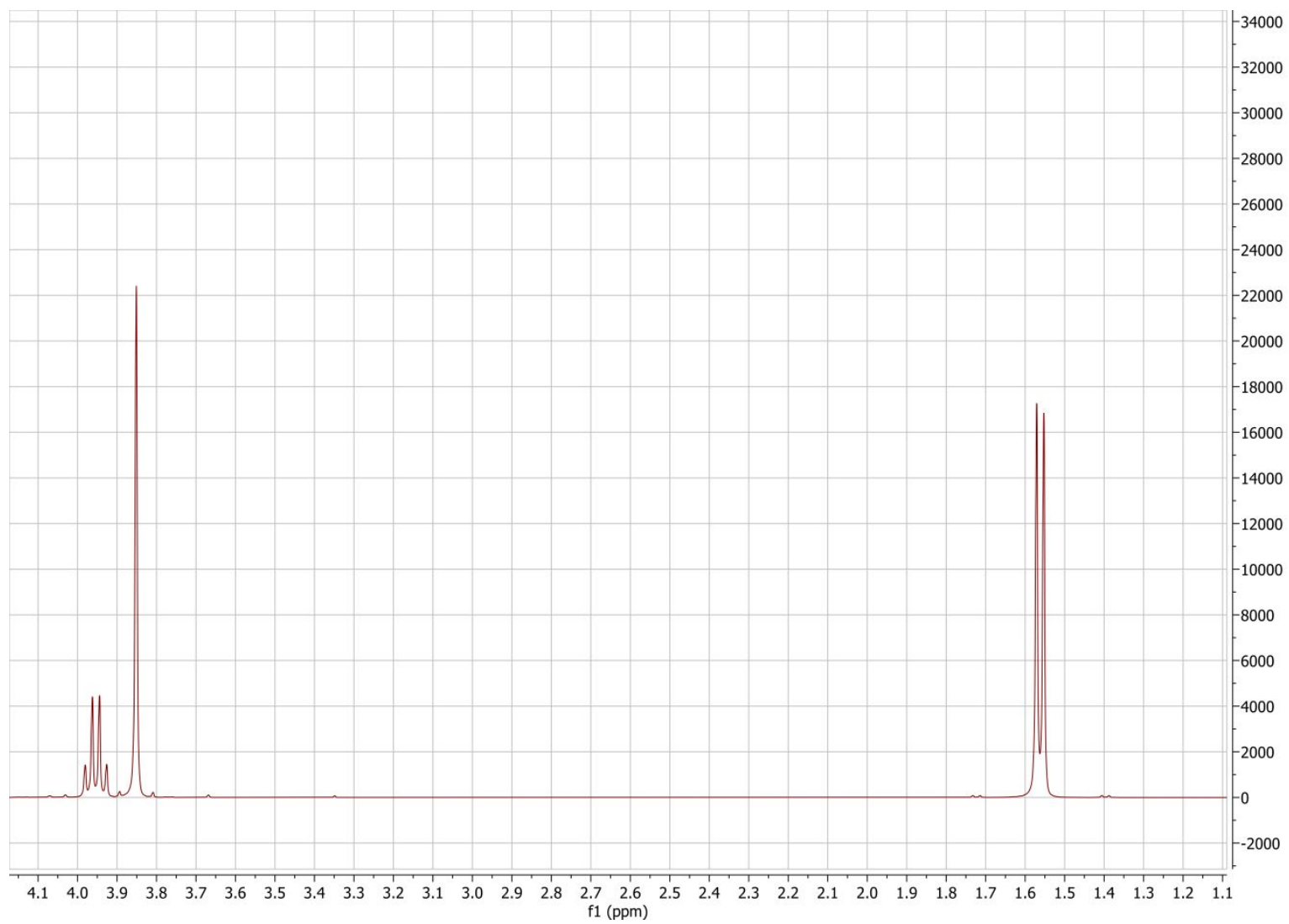


Figure S6 (Dirndorfer et al.)

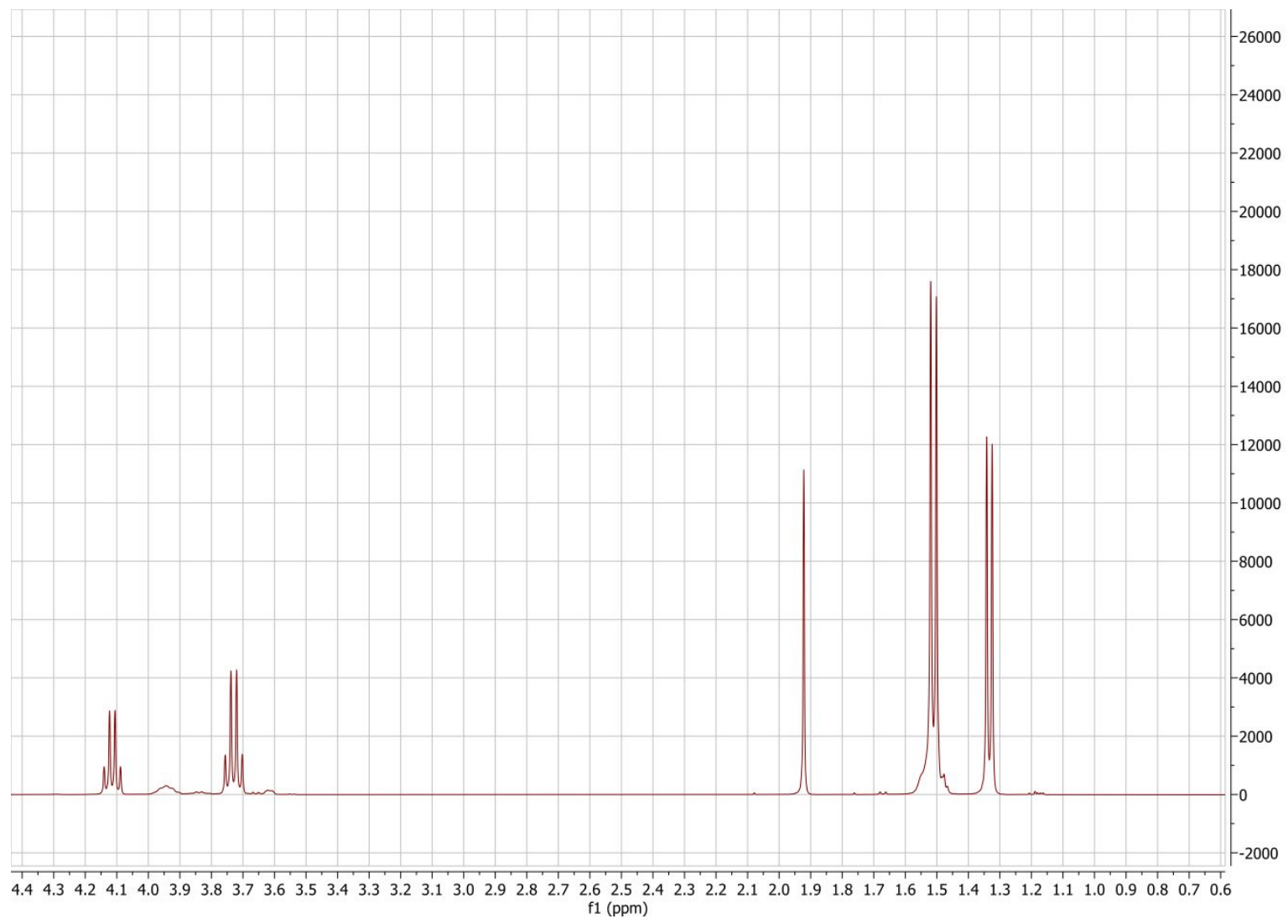


Figure S7 (Dirndorfer et al.)

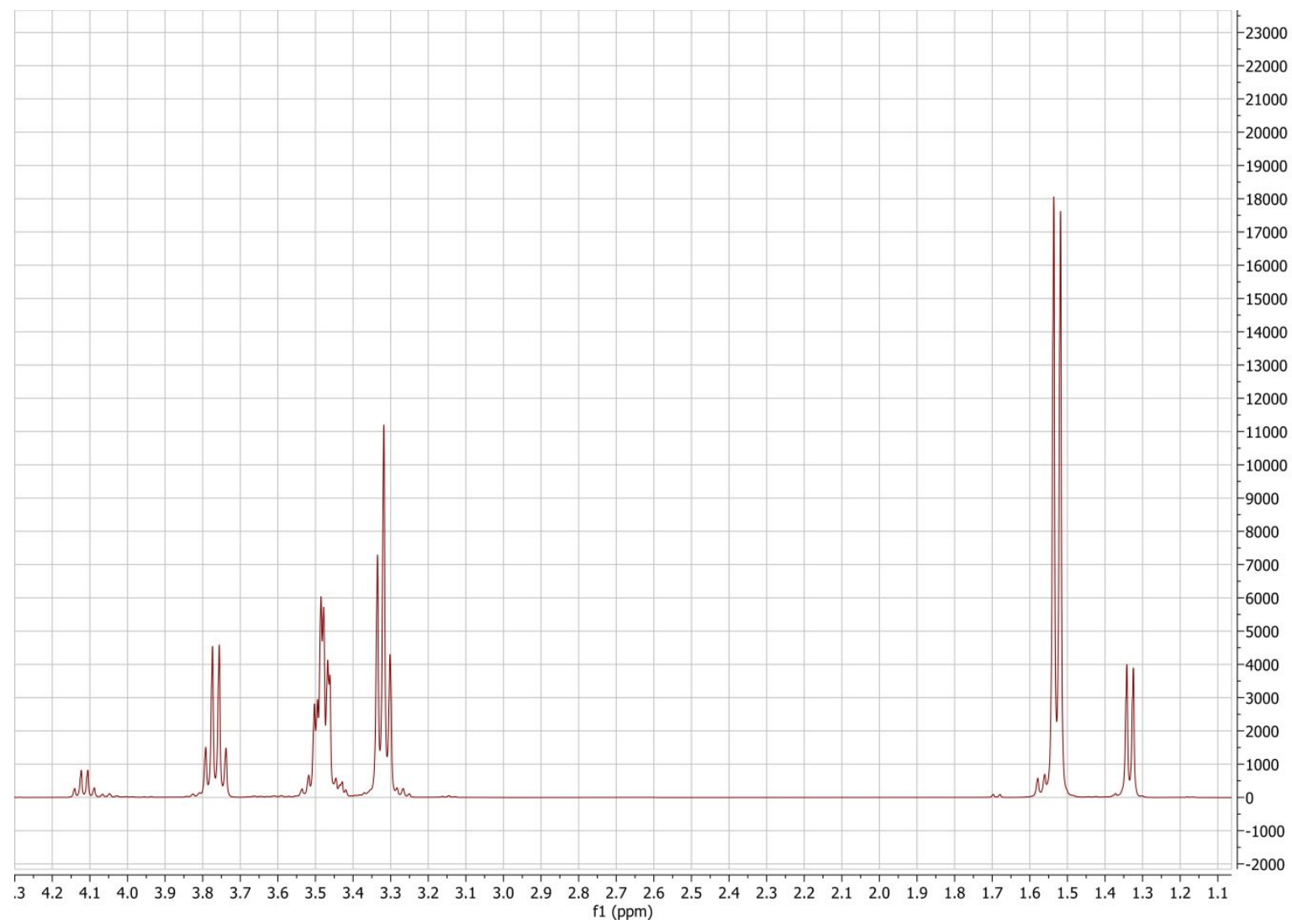


Figure S8 (Dirndorfer et al.)
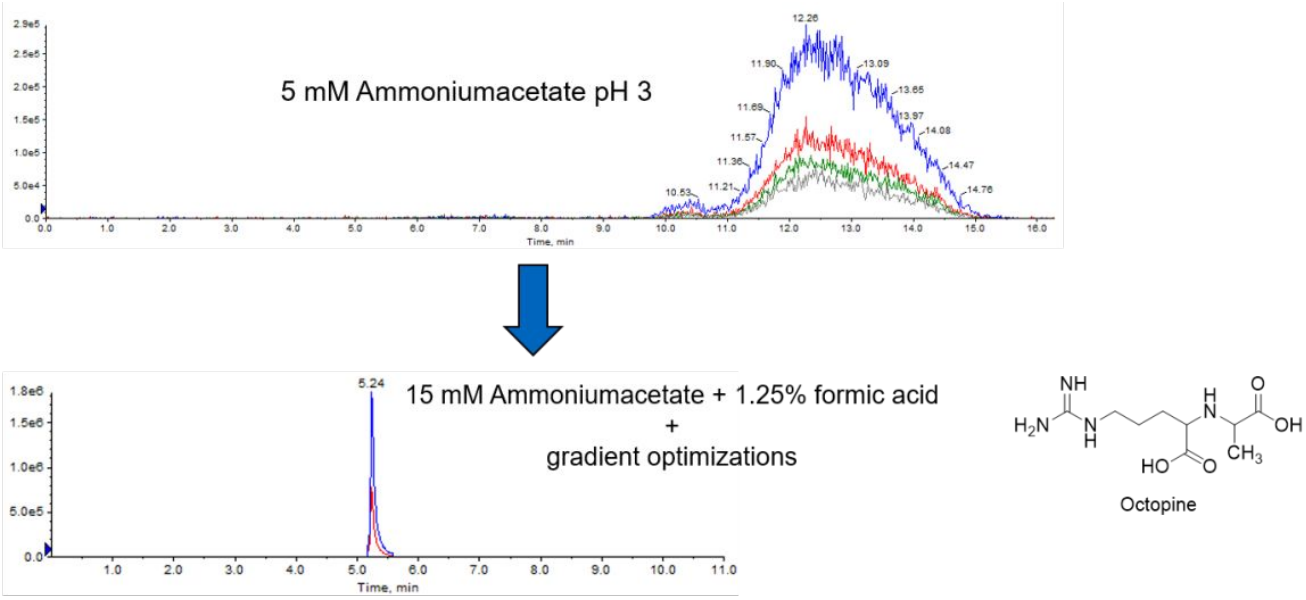

LC-MS/MS system: Shimadzu Nexera X2 with Sciex 6500 Qtrap

Stationary phase: Waters BEH Amide, $1.7 \mu \mathrm{m}, 2.1 \times 100 \mathrm{~mm}$

Flow rate: $\quad 0.5 \mathrm{~mL} / \mathrm{min}$

Injection volume: $\quad 1 \mu \mathrm{L}$

Mobile Phase: $\quad$ A: 15 mM Ammoniumacetate $+1.25 \%$ FA in $\mathrm{H}_{2} \mathrm{O}$

B: $15 \mathrm{mM}$ Ammoniumacetate + $1.25 \% \mathrm{FA}$ in $\mathrm{ACN} / \mathrm{H}_{2} \mathrm{O}(95 / 5)$

$\begin{array}{llll}\text { Gradient: } & \text { Time }[\mathrm{min}] & \mathrm{A}[\%] & \mathrm{B}[\%] \\ & 0 & 5 & 95 \\ & 1.5 & 5 & 95 \\ 3.5 & 27.5 & 72.5 \\ 5 & 55 & 45 \\ & 5.75 & 70 & 30 \\ 6.5 & 70 & 30 \\ 7 & 5 & 95 \\ 9 & 5 & 95\end{array}$

68 
Table S1. MRM transitions of unlabeled and stable isotope labeled opines (ESI ${ }^{+}$mode).

\begin{tabular}{|c|c|c|c|c|c|c|c|}
\hline Analyte (number) quant./qual. & $\begin{array}{c}\text { Q1 } \\
{[\mathrm{m} / \mathrm{z}]}\end{array}$ & $\begin{array}{c}\text { Q3 } \\
{[\mathrm{m} / \mathrm{z}]}\end{array}$ & $\begin{array}{c}\mathbf{t R} \\
{[\mathrm{min}]}\end{array}$ & $\begin{array}{l}\text { DP } \\
\text { [V] }\end{array}$ & $\begin{array}{l}\text { EP } \\
\text { [V] }\end{array}$ & $\begin{array}{l}\mathrm{CE} \\
{[\mathrm{V}]}\end{array}$ & $\begin{array}{l}\text { CXP } \\
\text { [V] }\end{array}$ \\
\hline Octopine (1) quant. & 247.1 & 142.0 & 4.7 & 51 & 10 & 29 & 16 \\
\hline Octopine (1) qual. & 247.1 & 130.0 & 4.7 & 51 & 10 & 27 & 14 \\
\hline (IS) Octopine- ${ }^{13} C_{6}\left(1-{ }^{13} C_{6}\right)$ quant. & 253.1 & 145.9 & 4.7 & 36 & 10 & 31 & 14 \\
\hline (IS) Octopine- ${ }^{13} C_{6}\left(1-{ }^{13} C_{6}\right)$ qual. & 253.1 & 101.9 & 4.7 & 36 & 10 & 33 & 10 \\
\hline Alanopine (2) quant. & 162.0 & 116.0 & 4.2 & 1 & 10 & 15 & 14 \\
\hline Alanopine (2) qual. & 162.0 & 70.1 & 4.2 & 1 & 10 & 27 & 8 \\
\hline (IS) Alanopine- ${ }^{13} \mathrm{C}_{3}\left(2-{ }^{13} \mathrm{C}_{3}\right)$ quant. & 165.1 & 117.9 & 4.2 & 31 & 10 & 15 & 12 \\
\hline (IS) Alanopine- ${ }^{13} C_{3}\left(2-{ }^{13} C_{3}\right)$ qual. & 165.1 & 71.9 & 4.2 & 31 & 10 & 31 & 8 \\
\hline Strombine (3) quant. & 148.0 & 102.0 & 4.4 & 1 & 10 & 13 & 12 \\
\hline Strombine (3) qual. & 148.0 & 56.0 & 4.4 & 1 & 10 & 27 & 8 \\
\hline Strombine- $d_{2}\left(\mathbf{3}-d_{2}\right)$ quant. & 150.0 & 103.9 & 4.4 & 26 & 10 & 13 & 12 \\
\hline Strombine- $d_{2}\left(3-d_{2}\right)$ qual. & 150.0 & 57.9 & 4.4 & 26 & 10 & 29 & 30 \\
\hline Tauropine (4) quant. & 198.0 & 152.0 & 4.2 & 46 & 10 & 17 & 8 \\
\hline Tauropine (4) qual. & 198.0 & 108.1 & 4.2 & 46 & 10 & 27 & 14 \\
\hline
\end{tabular}

